\title{
The Use of Ambrosia Moritima Methanolic Extract as Nephroprotective against Rifampin Induced Nephrotoxicity in Rats
}

\author{
Tarig HA Bilal ${ }^{1}$, Esraa Ramadan ${ }^{1}$, Idris OF $^{2}$, Sara A Mohamed*3 and Samia H Abdelrahman ${ }^{3}$ \\ ${ }^{1}$ Department of Biochemistry, School of Medical Laboratories Sharg Alneel College, Sudan
}

${ }^{2}$ Department of Biochemistry, Faculty of Science and technology, Alneelain University, Suda

${ }^{3}$ Department of Biochemistry, Veterinary Research Institute, Sudan

*Corresponding author: Sara A Mohamed, Department of Biochemistry, Veterinary Research Institute, Sudan.

To Cite This Article: Sara A Mohamed. The Use of Ambrosia Moritima Methanolic Extract as Nephroprotective against Rifampin Induced Nephrotoxicity in Rats. Am J Biomed Sci \& Res. 2019 - 5(3). AJBSR.MS.ID.000919. DOI: 10.34297/AJBSR.2019.05.000919.

Received: 眥 September 10, 2019; Published: 此 September 26, 2019

\begin{abstract}
Background and Objectives: Nephrotoxicity is a progressive loss in kidney function over a period of months or years. Rifampicin (RIF) is the first line drug for the treatment of tuberculosis and can cause nephrotoxicity in human beings and animals. Material and methods: Nephroprotective effect of methanolic extract of, Ambrosia moritima leaves was studied in healthy albino rats (1.25-1.75 kg) of either sex. This plant is a flowering plant in the family Asteraceae, they are distributed in the tropical and subtropical regions. It is used in traditional medicine for the treatment of renal failure, inflammation of kidney and other diseases. The renal toxicity was produced by administration of rifampin at dose rate of $70 \mathrm{mg} / \mathrm{kg} \mathrm{I} / \mathrm{p}$ for 28 days. The plant extract was given by simultaneous oral administration of methanolic extract of Ambrosia moritima leaves at a dose rate of 300 and $600 \mathrm{mg} / \mathrm{kg}$ for 28 days. Results: It was found that the induced toxicity was inhibited by the administration of the extract. The decreased levels of serum urea, creatinine, sodium ( $\mathrm{Na}$ ) and potassium (K). Histopathological examination revealed protection of the kidney from the marked necrosis of renal tubules that induced by rifampin. Conclusion: Ambrosia moritima methanolic extract has a marked nephroprotective effect depending on the concentration.
\end{abstract}

Keywords: Ambrosia moritima; nephroprotective activity; Rifampicin; Urea; Creatinine

\section{Introduction}

Plants are the most important source for traditional medicines and may contribute to the development of new medicines for various human health problems. Medicinal plants have played an essential role in the development of human culture, religions and ceremonies. $80 \%$ of the world's inhabitants rely mainly on traditional medicines for their primary health care [1]. Medicinal plants are most important to daily health and practices particularly in developing countries, also herbal medicine became a topic of augmented global importance having impacted on both world health and International trade [2]. Drugs are sometimes associated with side effects, whereas phytochemical (plant extract) have been found to have fewer side effects, better patient tolerance, relatively less expenses and a long history of use and renewability in nature.The kidneys are a pair of organs located in the back of the abdomen. Each kidney is about 4 or 5 inches long about the size of fist. The kidneys' functions are to filter the blood. All the blood in our bodies passes through the kidneys several times a day. The kidneys remove wastes, control the body's fluid balance, and regulate the balance of electrolytes [3]. Nephrotoxicity is poisonous effect of some substances, both toxic chemicals and medication on the kidneys. Many of the drugs causes toxicity to the kidney, these drugs used in chemotherapy such as Cisplatin, Carboplatin.

Varieties of natural products, medicinal plants and dietary components have been evaluated as nephroprotective agents. Currently, medicinal plants are widely used in the treatment of many diseases owing their safety, cheapness and nontoxicity when compared with the synthetic drugs [4]. Increasing health concerns necessitate reliance on alternate remedies for sustaining good health. Medicinal plants are extensively used for the management and treatment of various diseases. Plant drugs are considered nontoxic and devoid of side effects [5].

\section{Plant Material}

Ambrosia moritima leaves belong to the family of Asteraceae were collected in May 2017 from Alkhartoum city. The plant was 
authenticated by the botanists in medicinal and aromatic plants research institute.

\section{Preparation of extract}

$500 \mathrm{~g}$ of Ambrosia moritima was successively extracted by soaking in $80 \%$ methanol for about seventy-two hours of solvent with daily filtration and evaporation. Solvents were evaporated under reduced pressure to dryness using rotary evaporator apparatus.

\section{Experimental Design}

Group A: serving as control for 28 days. Group B: was injected by rifampin I/p- $0.8 \mathrm{ml} / \mathrm{Kg}$ induced nephrotoxicity for 28 days.
Group C: was injected by rifampin I/p-0.8 ml/Kg and treated with the low dose $(300 \mathrm{mg} / \mathrm{Kg})$ of the methanolic extract of Ambrosia moritima leaves. Group D: was injected by rifampin I/p-s $0.8 \mathrm{ml} /$ $\mathrm{Kg}$ and treated with high dose $(600 \mathrm{mg} / \mathrm{Kg})$ of methanolic extract of Ambrosia moritima leaves. Serum concentration of urea, creatinine, and electrolytes are measured using commercial kits (Biosystemm S.A Costa Barva 30, Barcelona Spain).

\section{Statistical Analysis}

Data were expressed as mean \pm standard error of mean (SEM). Statistical evaluation was done using SPSS (version 16.0).

\section{Results}

Table 1: Effect of Ambrosia moritima extract administered with Rifampin in the concentration of Serum Urea.

\begin{tabular}{|c|c|c|c|c|c|}
\hline Groups & Urea in zero Time & Urea in 7 Days & Urea in 14 Days & Urea in 21 Days & Urea in 28 Days \\
\hline Group A & $26.02 \pm 2.08$ & $27.01 \pm 4.11$ & $28.23 \pm 5.24$ & $29.62 \pm 5.81$ & $29.22 \pm 3.46$ \\
\hline Drug & $26.00 \pm 2.08$ & $41.21 \pm 6.11$ & $74.23 \pm 6.36$ & $84.47 \pm 5.81$ & $94.08 \pm 3.46^{*}$ \\
\hline Low dose & $20.62 \pm 1.20$ & $38.64 \pm 2.33$ & $60.33 \pm 3.18$ & $57.67 \pm 1.45 *$ & $57.00 \pm 1.53$ \\
\hline High dose & $22.24 \pm 1.06$ & $42.00 \pm 3.06$ & $64.08 \pm 2.28$ & $56.34 \pm .08$ & $53.23 \pm 1.24$ \\
\hline \multicolumn{6}{|c|}{ Values are means \pm Standard deviation } \\
\hline
\end{tabular}

Table 2: Effect of Ambrosia moritima extract administered with Rifampin in the concentration of Serum Creatinine.

\begin{tabular}{|c|c|c|c|c|c|}
\hline Groups & Creatinine zero time & Creatinine 7 days & Creatinine 14 days & Creatinine 21 days & Creatinine 28 days \\
\hline Group A & $0.64 \pm 0.07$ & $0.65 \pm 0.2$ & $0.68 \pm 0.24$ & $0.87 \pm 0.12$ & $1.25 \pm 0.22$ \\
\hline Drug & $0.71 \pm 0.07$ & $2.83 \pm 0.42$ & $3.88 \pm 0.23$ & $4.68 \pm 0.2$ & $5.48 \pm 0.32 *$ \\
\hline Low dose & $0.56 \pm 0.03$ & $0.74 \pm 0.4$ & $1.88 \pm 0.15$ & $1.84 \pm 0.07$ & $1.68 \pm 0.08 *$ \\
\hline High dose & $0.62 \pm 0.3$ & $1.63 \pm 0.2$ & $1.82 \pm 0.12 *$ & $1.88 \pm 0.10$ & $1.54 \pm 0.04$ \\
\hline \multicolumn{6}{|c|}{$\begin{array}{l}\text { Values are means } \pm \text { Standard deviation } \\
* \text { significant }\end{array}$} \\
\hline
\end{tabular}

We designed the current study to test the hypothesis that Am- brosia moritimam leaves for 28 days induced significant $(\mathrm{P}<0.05)$ brosia moritimam leaves extract could protect against Rifampin decreases in high serum levels of urea, creatinine (Table 1,2). The (RIF)-induced renal damage in rats through attenuation of inflam- concentration os sodium and potassium were increased when the mation and prevention of oxidative stress. Oral administration of rifampin was injected to the rats, administration of A. moritimam rifampin in dose of $70 \mathrm{mg} / \mathrm{kg} \mathrm{I} / \mathrm{p}$ for 28 days to rats caused nephro- leaves extract resulted in decreased level of both sodium and potastoxicity manifested by significant increase $(\mathrm{P}<0.05)$ in serum level sium, however the decrease was marked with the high dose when of urea, creatinine, $\mathrm{Na}$ and $\mathrm{K}$ when compared with the (negative) compared with rifampin intoxicated rats (Table 3,4). control group. Oral administration of methanolic extract of Am-

Table 3: Effect of Ambrosia moritima extract administered with Rifampin in the level of Sodium.

\begin{tabular}{|c|c|c|c|c|c|}
\hline Groups & Na zero time & Na 7 days & Na 14 days & Na 21 days & Na 28 days \\
\hline Group A & $128.02 \pm 0.52$ & $138.67 \pm 1.2$ & $136.56 \pm 1.21$ & $136.76 \pm 2.22$ & $136.02 \pm 1.15$ \\
\hline Drug & $138.21 \pm 0.32$ & $136.67 \pm 1.2$ & $142.44 \pm 1.32$ & $148.42 \pm 2.12$ & $154.24 \pm 1.15 *$ \\
\hline Low dose & $134.00 \pm 1.44$ & $146.23 \pm 0.8$ & $152.42 \pm 1.08$ & $146.53 \pm 0.44$ & $146.02 \pm 1.53 *$ \\
\hline High dose & $135.27 \pm 0.48$ & $148.43 \pm 1.2$ & $148.04 \pm 1.08$ & $148.22 \pm 0.8^{*}$ & $164.02 \pm 0.02$ \\
\hline \multicolumn{6}{|c|}{$\begin{array}{l}\text { Values are means } \pm \text { Standard deviation } \\
\qquad * \text { significant }\end{array}$} \\
\hline
\end{tabular}

Table 4: Effect of Ambrosia moritima extract administered with Rifampin in the level of Sodium.

\begin{tabular}{|c|c|c|c|c|c|}
\hline Groups & K zero time & K 7 days & K 14 days & K 21 days & $4.08 \pm 0.08$ \\
\hline Group A & $3.80 \pm .12$ & $3.82 \pm 0.02$ & $4.24 \pm 0.04$ & $6.03 \pm 0.09$ \\
\hline Drug & $3.56 \pm .09$ & $3.64 \pm 0.02$ & $4.43 \pm 0.08$ & $4.97 \pm 0.08$ \\
\hline
\end{tabular}




\begin{tabular}{|c|c|c|c|c|c|}
\hline Low dose & $3.68 \pm .12$ & $4.32 \pm 0.12$ & $5.32 \pm 0.20$ & $5.24 \pm 0.12$ & $5.47 \pm 0.12 *$ \\
\hline High dose & $3.54 \pm .08$ & $4.42 \pm 0.21$ & $5.46 \pm 0.21$ & $5.20 \pm 0.12$ & $6.60 \pm .06 *$ \\
\hline \multicolumn{5}{|c|}{$\begin{array}{c}\text { Values are means } \pm \text { Standard deviation } \\
\text { significant }\end{array}$} \\
\hline
\end{tabular}

\section{Discussion}

It has been reported that nephropathy is an important microvascular complication of anti-tuberculosis therapy multiple studies have reported that oxidative stress and inflammation are implicated in drug-induced organ toxicity. However, renal injury induced by anti-tuberculosis therapy is usually reversible and could be treated appropriately if detected early. Therefore, the use of antioxidants could protect against RIF-induced oxidative stress and nephrotoxicity. The results obtained showed that the administration of Rifampin (RIF) has induced renal injury and glomerular dysfunction as evident by the elevated serum urea, creatinine, sodium and potassium levels. These parameters are often regarded as reliable markers of renal damage [6]. In addition, serum creatinine has been used to estimate glomerular function and its elevation is an indicator of renal failure [7]. The elevated serum markers of renal toxicity is agreement with the study of Hashmi et al. [3], who reported increased serum levels of urea and creatinine in albino rabbits following administration of anti-tuberculosis drugs Isoniazid (INH) and Rifampin (RIF) induced nephrotoxicity. In the present study, results suggested that methanolic extract of Ambrosia moritimam $600 \mathrm{mg} /$ kg significantly reduced Rifampin-induced elevated serum levels of creatinine, urea, sodium, and potassium. The histological findings also supported the nephroprotective action of methanolic extract of Ambrosia moritimam.

\section{Conclusion}

It can be concluded that Ambrosia moritimam has anaphor protective effect which might be due to the presence of one or more of flavonoids. In experimental animals several strategies to ameliorate the toxicity have been attempted. These include controlling the time of administration of the antibiotics, modifying the diet and co administering agent to mitigate the renal toxicity.

\section{References}

1. kidney Disease Improving Global Outcome (2009) KDIGO, Clinical Guideline for kidney Disease-Mineral and Bone Disorder. (CDK-MBD) Kidney Int 76 (Suppl 113).

2. Ansel Howard C, Allen Loyd V Jr, Popovich Nicolas G (2000) Pharmaceutical Dosage forms and drug delivery systems. $\left(7^{\text {th }}\right.$ edn), Lippincott Williams and Wilkins (Pub.), USA.

3. Hashmi N, Muhammad F, Javed I (2013) Nephroprotective effects of Ficusreligiosalinn (peepals plant) stem bark against isoniazid and rifampicin induced nephrotoxicity in albino rabbits. Pak Vet J 33(3): 330-334.

4. Ogbera AO, Dada O, Adeyeye F, Jewo PI (2010) Complementary and alternative medicine use in diabetes mellitus. West Afr J Med 29(3): 158-162.

5. Bhawna S, SU Kumar (2009) Hepatoprotective activity of some indigenous plants. Int J Pharm Tech Res 1: 1330-1334.

6. Adebisi SA, Oluboyo PO, Okesina AB (2000) Effect of drug-induced hyperuricaemia on renal function in Nigerians with pulmonary tuberculosis. Afr J Med MedSci 29 (3-4): 297-300.

7. Adeney AA, Benebo AS (2008) Protective effect of the aqueous leaf and seed extract of Phyllanthusamarus on gentamicin and acetaminophen-induced nephrotoxicity in rats. J Ethnopharmacol 118(2): 318323. 